\title{
Comparison of sweat bottle and sweat print measures under resting-state conditions*
}

\author{
ROBERT STRAHAN $\dagger$ and JOHN TODD \\ University of Rochester, Rochester, New York 14627 \\ and \\ JOHN CONNOLLY $+\dagger$ \\ St. John Fisher College, Rochester, New York 14618
}

\begin{abstract}
Palmar sweat measurements were obtained under resting-state conditions using both the newly developed sweat bottle technique and the frequently employed sweat print method. Measurement reliability was high for both approaches. The two methods, at least under generally nonstressful conditions, appear to reflect essentially different aspects of palmar sweating.
\end{abstract}

A new palmar sweat measure has been described (Strahan, Todd, \& Inglis, in press) the administrative ease of which holds special promise for naturalistic or field assessment. In the sweat bottle technique, a sweat sample is obtained by inverting a small bottle of distilled water over a fingertip or palm for a few seconds. The more sweat entering the bottle, the more ions; conductivity of the resulting solution can later be determined with simple, relatively inexpensive apparatus. Extensive reliability data have been gathered for this sweat measure, and some validational data as well.

The sweat print technique is perhaps most of ten used for the measurement of palmar sweating. Even before Silverman and Powell's (1942) influential work, several versions of this technique had been suggested. Briefly and generally, a chemical solution is applied to a fingertip and allowed to dry for a short period (a minute or so). A piece of chemically treated paper is then affixed to the fingertip for another few minutes. The ensuing reaction, resulting from juxtaposition of the two sets of chemicals and the sweat intermediary, darkens the sweat band, with judgments of the degree of darkening then serving as indices of the degree of sweating.

The present study investigated the relation of the new sweat bottle measure to a measure of the established sweat print.

\section{METHOD}

\section{Subjects}

Twenty-six students from an introductory psychology course served as Ss in order to meet part of a research participation requirement.

\footnotetext{
*This research was largely conducted when the third author was an NSF Undergraduate Research Participant (NSF Grant GY-9858) at the University of Rochester.

†Now at Iowa State University. Address reprint requests to Robert Strahan, Department of Psychology, Old Botany Hall, Iowa State University, Ames, Iowa 50010.

t+Now at the State University of New York at Plattsburgh.
}

\section{Sweat Measures}

Three sweat bottle measures were employed: A palm sweat bottle (PSB), a heel sweat bottle (HSB), and a finger sweat bottle (FSB). In the first case (PSB), a 120 -ce polyethylene bottle with a neck inner diameter of $17 \mathrm{~mm}$ was applied to the center of a palm; in the second case (HSB), an identical bottle was applied to the heel of a hand, the fleshy part opposite the thumb; in the third instance (FSB), a bottle with a neck inner diameter of $12 \mathrm{~mm}^{1}$ was applied successively to the tips of the middle finger, ring finger, and little finger of the same hand. In all cases, $30 \mathrm{cc}$ of distilled water were used, bottle application was for $5 \mathrm{sec}$, the bottle was shaken gently during application, and residual drops were collected on the bottle's removal with a slight scraping motion.

PSB and HSB were applied directly to unprepared skin. FSB was employed after skin washing, specifically, $3 \mathrm{~min}$ af ter the finger- and thumbtips had been rubbed together by the $S$ in a basin of water, then dried. The sweat collected by FSB was thereby restricted to that secreted during the 3-min waiting period. Reciprocals of all sweat conductivity measurements permitted expression in units of electrical conductance.

The finger sweat print (FSP) procedure was essentially that described by McNair, Droppleman, and Kussman (1967). After application of the ferric chloride solution to the tip of a forefinger, $1 \mathrm{~min}$ elapsed before a coated sweat band was at tached firmly with a cellophane tape; 2 min later, the band was removed. Independent, blind ${ }^{2}$ ratings of the FSPs were performed by the three investigators using the 1-to-10 visual rating procedure of Kenyon and Müller (1963).

\section{Order of Administration}

Each of the four sweat measures was applied to both hands, more or less simultaneously. First step in the procedure was the coating of the forefingertips with the ferric chloride solution. Washing of the remaining fingertips then commenced, timed so that drying would be complete just at the end of the 1 -min FSP waiting period. FSP bands were then attached, first to the left, then to the right forefingers. Two minutes later, the bands were removed in this same left-to-right order.

Another minute later the FSBs were applied, in middle-to-little finger sequence; left- and right-hand applications were made at the same time. Immediately following, PSB and HSB sampling was undertaken, in each case simultaneously for left and right hands; order was alternated for Ss, half receiving PSB first.

The same $f$ gathered all swe at measure data. 
Table 1

Intercorrelations and Alternate-Form Reliabilities of the Sweat Measures

\begin{tabular}{lcccc}
\hline & PSB & HSB & FSB & FSP \\
\hline PSB & $(.86)$ & .75 & .53 & .29 \\
HSB & & $(.91)$ & .69 & .13 \\
FSB & & & $(.96)$ & .25 \\
FSP & & & & $(.86)$ \\
\hline
\end{tabular}

Note-r.05 $=.39, r_{.01}=.50, r_{.001}=.61$

\section{RESULTS}

Interrater correlations were high for the 52 FSP ratings: $.92, .91, .93$; the three ratings for each FSP were then averaged for still greater reliability.

For all sweat measures, left- and right-hand readings were averaged to simplify data analysis and to provide more consistent measurement. The parenthesized coefficients in Table 1 are right-hand/left-hand correlations for each sweat variable, corrected via the Spearman-Brown formula for greater comparability with the other correlations (involving, as noted, combined-hand scores). Clearly, all four measures exhibited very adequate, and nearly equal, alternate-form (-hand) reliabilities.

The three sweat bottle measures (again Table 1) were substantially related to one another, but at levels quite below their reliabilities. It was not surprising to find the highest correlation between PSB and HSB, since these two measures were alike in their application to unwashed skin. In testing for significance between the two correlated (same Ss) correlations (Guilford, 1965), PSB's association with HSB was seen to be significantly greater than its association with FSB $\left(\mathrm{t}_{23}=2.12, \mathrm{p}<.05\right)$; the difference between the two correlations involving HSB (i.e., those with PSB and FSB) was not significant $\left(\mathrm{t}_{23}=\right.$ 0.58 ).

With respect to the major question of the study, there was little evidence for a sweat-bottle/sweat-print relation. Though all three correlations with FSP were positive, as expected, none approached conventional significance levels. The relatively small sample size precludes the inference of near-zero or trivial relation. It is clear, however, that the sweat-bottle and sweat-print measures are by no means equivalent: upper bounds of the $95 \%$ confidence intervals for the three FSP correlations were $.61, .49$, and .58 . Correction for attenuation due to unreliability increased these bounds only slightly, because of the high values of the reliability estimates.

The distributions of the variou measures are of some interest. Though all four were roughly symmetric and unimodal, some degree of positive skew was seen for the sweat bottle variables, particularly FSB. Consequently, the correlational analyses were repeated using the skew-reducing natural logarithms $f$ these variables. The new coefficients were very like the originals based on raw scores.

Another supplementary analysis dealt with potential differences between palm- and heel-sweat levels. The palm is a relatively protected part of the hand, and thus would seem less susceptible to error through the inadvertent wiping away of sweat prior to measurement; it would also seem to offer a more uniform focus for bottle application than the heel. On the other hand, the palm's sweat gland density appears to be less than that of the heel (Kuno, 1956). A comparison of means confirmed this hypothesis of greater heel sensitivity: $\mathrm{t}_{25}$ $=3.58, \mathrm{p}<.01$. The two techniques seem about equally reliable (Table 1), though this equality might not hold for less well controlled, nonlaboratory studies.

\section{DISCUSSION}

The slight sweat-bottle/sweat-print relations here reported might be compared with those obtained in a pilot study $(n=25)$ involving HSB, FSB, and FSP measures. When the bottle-print correlations from these two studies were averaged (Fisher transformation, with appropriate weighting by respective degrees of freedom), results were as follows. For both HSB-FSP and FSB-FSP associations, the correlation coefficient was .31 $(\mathrm{p}<.05)$ : significant support for sweat-bottle/sweat-print association, but support suggestive of a weak relationship.

It is important, however, to note that all sweat measurements were taken under ordinary resting-state conditions. Though some Ss may have experienced a degree of arousal or tension in response to the novelty and unknown nature of the experiment, no obvious stressor was employed. The correlations obtained, then, represent associations under generally benign conditions and may not be typical of those to be seen in arousing or emotional circumstances. One might, e.g., expect greater independence between sweat bottle readings under washed and unwashed conditions when recording takes place during changing arousal. This expectation would follow because washed measurements are sensitive just to transient sweating, whereas unwashed ones reflect residual (prior state) levels as well. And one might not be surprised to observe stronger sweat-bottle/sweat-print associations with presentation of a stressor whose potency across Ss is variable but, on average, moderately great.

Previous work (Strahan et al, in press) comparing sweat bot tle measures to another, presumably rejated variable, skin conductance, has yielded equivocal results. In one study, a very high and quite reliable correlation was found: $r=.93(n=13$, $\mathrm{p}<.001)$. In another $(\mathrm{n}=24)$ investigation, small and nonsignificant coefficients resulted. In this latter study, however, both sweat bottle and skin conductance variables exhibited similar (and statistically significant) responses to the presentation, then to the removal of a stressor stimulus (a shock-paced mental arithmetic task).

Overall, the main implication of the present work seems to be this: That whereas both sweat bottle and sweat print methods reliably measure certain aspects of palmar sweating, those aspects are by no means identical, and may be quite distinct-at least in a relatively benign context such as that of this inquiry. Given the administrative advantages of the sweat bottle technique, together with previous reliability and validity data speaking to its psychological usefulness, more extensive study of this measure would seem worthwhile.

\section{REFERENCES}

Guilford, J. P. Fundamental siatistics in psychology and 
education. (4th ed.) New York: McGraw-Hill, 1965.

Kenyon, J., \& Müller, H. F. A visual rating scale for prints of finger-sweat. American Journal of Psychology, 1963, 76, 140-142.

Kuno, Y. Human perspiration. Springfield, Illinois: Thomas, 1956.

McNair, D. M., Droppleman, L. F., \& Kussman, M. Finger sweat print tape bands. Psychophy siology, 1967, 4, 75-78.

Silverman, J. J., \& Powell, V. E. Studies on palmar sweating: I. A technique for the study of palmar sweating. American Journal of Medical Science, 1942, 208, 297-305.

Strahan, R. F., Todd, J. B., \& Inglis, G. B. A palmar sweat measure particularly suited for naturalistic research. Psychoph y siology, in press.

\section{NOTES}

1. This is about the largest diameter that will fit a fingertip. The palm and heel can of course accommodate larger sizes, and 17-mm bottles were used there for their increased ion-collecting capacity.

2. All prints from both hands, arbitrarily coded, were shuifled together before rating.

(Received for publication October 5, 1973; revision received January $17,1974$. ) 